mination repeated, proving that the liquid was practically homogeneous. A determination of the density of the gas also gave results agreeing satisfactorily with the theory.

There seems, therefore, to be little question that the butane obtained by Professor Mabery was in reality normal butane, and that the boilingpoint of isobutane is $-11.5^{\circ}$.

W. A. NOYES.

The following note has been received from Prof. Mabery to whom this note was submitted in manuscript:

"I have no reason to doubt the accuracy of Professor Noyes' observation on the boiling-point of isobutane. It is not incompatible with our results on the butane in petroleum. I have intended to refer to this subject more fully in a later paper, a resume of the composition of American petroleum."

C. F. Mabery.

UNIVERSITY OF ILLINOIS, URBANA, ILL.

The Stereochemistry of Indigo.-The last paragraph of this paper (This Journal, December number, 1907, p.1 743,) in which the structures of the two diacetyl indigo whites are discussed on the spatial hypothesis, assumes that both compounds possess the ketone structure. If both rearrange to the tautomeric enol forms, it should be pointed out that there would still be two stereoisomers (cis and trans) which it would not be possible to resolve into optically active isomers. K. George Falk and J. M. Nelson.

\title{
REVIEW.
}

\section{RESEARCHES ON THE DENSITY OF GASES}

CARRIED ON DURING I904, I905 AND I906 IN THE PHYSICAL CHEMISTRY LABORATORY OF THE UNIVERSITY OF GENEVA. ${ }^{1}$

BY PEILIPPE A. GUYE.

Received October I, I907.

The present article contains a résumé of the results obtained during the course of three years' work on the exact density of gases. The work has been carried on in collaboration with Messrs. Jaquerod, Pintza, Davila, Gazarian and Baume, and until now has been the subject of only isolated publication (Jaquerod and Pintza, Compt.rend., 139, I29 (I904), ( $\mathrm{SO}_{2}$ and $\mathrm{O}_{2}$ ); Guye and Pintza, Ibid., I39, 679 (I904); ז4I, 5I (I905), ( $\mathrm{N}_{2} \mathrm{O}$, $\mathrm{CO}_{2}$ and $\mathrm{NH}_{3}$ ); Guye and Davila, Ibid., I4I, 826 (I905), (NO); Guye and Gazarian, Ibid., 143, I233 (I906), (HCl); Baume, unpublished (I907), $\left(\mathrm{SO}_{2}\right)$ ). These have contributed to the problem of the physicochemical determination of exact molecular weights, with a view to checking up the

${ }^{1}$ From Archives des Sciences Physiques et Naturelles, 24, 32-62. Translated by Helen Isham. 
atomic weights. This problem has become one of great importance, and has been taken up by Leduc (Leduc: Recherches sur les gaz. Paris, Gat1thierVillars (in part), Ann. Chim. Phys. (1897)), Lord Rayleigh (Rayleigh, Proc. Roy. Soc., 43, 353 (1 888), ( $\mathrm{H}_{2}$ and $\left.\mathrm{O}_{2}\right) ; 50,449$ ( 1892$),\left(\mathrm{H}_{2}\right.$ and $\left.\mathrm{O}_{2}\right) ; 53, \mathrm{I} 34$ (I893), $\left(\mathrm{O}_{2}, \mathrm{H}_{2}, \mathrm{~N}_{2}\right.$ atm. air); 55, 340 (I894), $\left(\mathrm{N}_{2}\right)$; 59, I98 (I896), (Ar, $\mathrm{He}) ; 62,204(\mathrm{I} 897),\left(\mathrm{CO}, \mathrm{CO}_{2}, \mathrm{~N}_{2} \mathrm{O}\right) ; 74$, I8I (1904), ( $\left.\mathrm{N}_{2} \mathrm{O}\right)$; Phil. Trans. Roy. Soc. 204A, 35 I (1905); Rayleigh and Ramsay, Phil. Trans. Roy. Soc. 186A, I87 (I895)), Morley, ( $Z$. physik. Chem., 20, I, $\left(\mathrm{O}_{2}\right)$; 22, 2, $\left(\mathrm{H}_{2}\right)$, (1896)), Gray, $\left(J\right.$. Chem. Soc., 87, I601 (1905), ( $\mathrm{N}_{2}$; $\left.\mathrm{O}_{2}, \mathrm{NO}\right)$; Proc. Chem. Soc. (1907), (HCl)), as well as Perman and Davies (Perman and Davies, Proc. Roy. Soc. 78A, 28 (1906)). Details of the methods, and the method of calculating the results, will be given under the head of generalities; next a review of the results obtained at Geneva compared with those of other experimenters, and finally a summary of those values which appear to the author to be most reliable.

\section{Generalities.}

Methods.--Two general methods have been used in density determinations, that of the balloon and that of the volumeter.

The balloon method, the details for which have been worked out by Regnault (weighing the balloon empty and filled with gas, against a counterpoise of the same volume, reducing to weight in vacuo, etc.) has been used in recent work, taking into account the correction for the contraction of the empty balloon, and the correction for the compressibility of the gas (deviation from the law of Mariotte between the existing pressure and that of $760 \mathrm{~mm}$. $\mathrm{Hg}$ ). 'The recent work is also characterized by a gradual reduction of the capacity of the balloon. Morley worked with balloons of from 8 to 2 I liters capacity. Rayleigh used one of about I.8 liters capacity, Leduc one of about 2.3 liters. The work in this laboratory has led to a still further reduction of the capacity of the balloon, and two balloons, destined to act as checks upon each other, one 0.8 liter, the other about 0.4 liter in capacity, filled at $0^{\circ}$ and under the same pressure conditions, have been used. ${ }^{1}$

Perman and Davies have used a balloon of 0.5 liter capacity, while Gray has reached the lowest unit, and in the determination of nitric oxide used one of about 0.267 liter. Contrary to what one might expect, a priori, the determinations with the small balloons are at least as concordant, among themselves, as those with balloons of larger volume. The experiments with the two-liter balloons below, show, on the whole, a better agreement than those with balloons varying from 8 to $2 \mathrm{I}$ liters. This may be due either to the fact that the corrections for the original weights (for contraction, etc.) are smaller with the small balloons, or that the chances of accidental error, especially those due to the condition of the surface of the glass, are diminished.

The only objection which can be raised to the use of the small balloons is the possibility of the condensation of gas on the inner wall. If there were such condensation, the density of gases measured in small balloons should be greater than in the larger balloons, and this error should be especially great in the case of the hygroscopic gases, sulphur dioxide, ammonia, hydrochloric acid gas, etc.

${ }^{1}$ Experiments using still smaller balloons are being carried on at present. 
The recent determinations show that while the capacity of the balloons varies to a great extent, the results obtained are very concordant, especially in the case of ammonia and sulphur dioxide.

By the volumeter method (capacity = about 1.8 liters) Perman and Davies found 0.77085 gram for the weight of a liter of ammonia gas under standard conditions, while by the balloon method they obtained 0.77086 gram for the same gas, using a balloon of 0.5 liter capacity. Guye and Pintza, using a volumeter of 3.5 liters capacity, found 0.7708 . Thus there is no appreciable difference between the values obtained by the large and small balloons. Jaquerod and Pintza have obtained the value 2.9266 grams for the weight of I liter of sulphur dioxide, using a volumeter of 3.5 liters capacity; Leduc, using a 2.3 liters balloon, found 2.9266; and Baume has recently repeated this determination, using two balloons of 0.53 liter and 0.32 liter capacity, respectively, on gas prepared under the same conditions as that used by Jaquerod and Pintza, and has found 2.9266 grams.

The only necessary precaution consists in rinsing the balloon, not only with air dried over phosphorus pentoxide, but also several times with the well-dried gas, the density of which is to be determined, taking care that between each rinsing operation the vacuum in the balloon is as perfect as possible, and that air does not enter the balloon between each determination.

It might, nevertheless, be assumed that there is a slight condensation of the gas on the inner wall of the glass, similar to the moisture adhering to glass. The author's experience (in collaboration with Gazarian) on the density of hydrochloric acid gas, which is extremely hygroscopic, does not justify this assumption. In fact the balloons were at first rinsed only two or three times with dry hydrochloric acid gas. Under these conditions, with a balloon of 0.385 liter capacity, the values for the density showed a regular decrease, and did not become constant until after the fourth determination. Using a larger balloon (o.818 liter capacity) this point was not reached after the seventh filling. These facts led to the conclusion that after twelve successive rinsings ${ }^{1}$ with the dry gas, each rinsing followed by a complete evacuation of the balloon, the condensation of the gas on the interior wall of the balloon is not sufficiently great to affect the results to an appreciable extent, provided air has not entered the balloon during the process of rinsing. (If Bunsen's observations are correct, the moisture given off by the glass at high temperatures is not due to condensation on the surface, but to water incorporated chemically or physically in the mass of the glass.) ${ }^{2}$

${ }^{2}$ In the case of non-hygroscopic gases 5 to 6 successive rinsings, each followed by complete evacuation, are sufficient.

${ }^{2}$ If we admit that the condensation of the gas on the interior surface of the balloon is not generally appreciable, still we do not claim that it is absolutely nil. In order to determine the condensation most accurately, two or more balloons of very different capacities should be filled at the same time, under the same conditions of pressure and with gas from the same source. The determinations of the densities of nitric oxide and sulphur dioxide, using two balloons filled simultaneously, furnish some preliminary information on this subject. The following averages have been 
The volumeter method, used as an exact method for the first time by Morley in the determination of the density of hydrogen, has been used in two ways. According to the first method the gas is evolved from an apparatus which may be weighed, and constructed in such a fashion that the gas alone, in a state of perfect purity and dryness, escapes. The gas then passes through a tight-fitting joint into a system of one or more balloons of known capacity, maintained at $o^{\circ}$, and which have been evacuated by a mercury pump. The pressure is measured either with a special manometer, or simply by connecting the apparatus with the tube of a barometer. Morley employed the latter method in his fourth series of measurements of hydrogen, and it has also been employed at Geneva. Knowing then the temperature and the pressure, the capacity of the balloons and the loss of weight of the generating apparatus, the density may be calculated.

According to the second method the volumeter, previously evacuated, is filled with the pure gas. The process of purifying may be more complete, as there is no limit to the weight of the apparatus used for that purpose. The volumeter being filled with the pure gas is held at $0^{\circ}$ and the exact pressure (about $760 \mathrm{~mm}$.) is read. Then the gas is absorbed in a suitable apparatus, previously evacuated and weighed, and connected to the volumeter by a tight-fitting joint.

The densities of hydrogen (Morley), oxygen and sulphur dioxide (Jaquerod and Pintza) have been determined by the first method, by the second those of nitrots oxide, carbon dioxide and ammonia (Guye and Pintza), and, more recently, ammonia (Perman and Davies).

The volumeter method used does not take into account the correction for the contraction of the balloons, but only that necessary for the reduction of the weights to weights in vacuo (for the evolution or absorption apparatus is weighed with a counterpoise of the same glass and the same volume) and finally the pressure coefficient of the gas in order to calculate the volume at a pressure of $760 \mathrm{~mm}$.

Whichever method is employed (balloon or volumeter), the correction for the pressure coefficient of the gas is negligible, since the pressure differs from $760 \mathrm{~mm}$. by only a few millimeters.

found for the weight of a liter of these gases, determined simultaneously with two balloons.

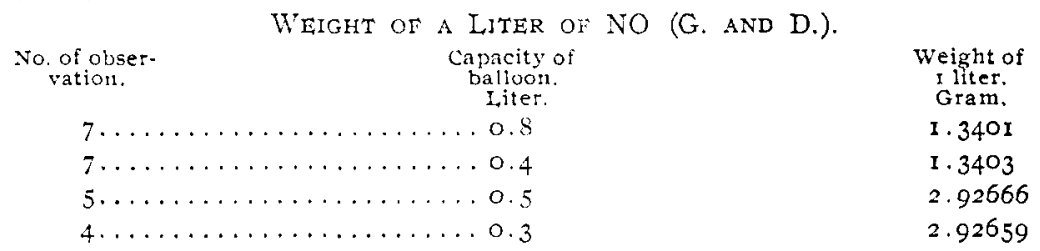

It may be claimed that this condensation was not on the surface of the glass, but in the special grease used to insure a tight cock. It may be well to cite here the recent work of Swinton (Chem. News, 95, I349 (1907)), who has shown that the gases, hydrogen and helium, have no chemical action on the surface of glass, and that the surface condensation is of a purely mechanical nature. The recent observations of Travers (Proc. Roy. Soc. 78A, 9 (1906)) on the condensation of gases by solid bodies should not be overlooked, if the density of a gas is to be determined with an accuracy exceeding I part in 10,000 . 
Choice of a Unit.-A common unit is indispensable. Leduc has reduced his values either to the density with regard to air, or to the weight of a liter of gas at Paris, or both. Morley expresses his results in weight per liter under standard conditions, that is, at $0^{\circ}$ and $760 \mathrm{~mm}$. pressure, at sea level and latitude $45^{\circ}$. Rayleigh calculates his results either to density compared with air or compared with oxygen (taken equal to 32) or as the weight of I liter at London or at Paris. At Geneva the weight of the liter under standard conditions has been directly determined. Gray, as well as Perman and Davies, has likewise used this unit, which is the unit for all results which are given in the following pages.

D. Berthelot (Compt. rend., I44, 269 (1907)) has recently calculated the greater number of measurements, comparing densities to that of oxygen. Instead of taking one value for oxygen, he adopts, in each case, the value obtained by each investigator for the density of this gas. This system presents several disadvantages: First, in the case of those determinations made by investigators who have not determined the value for oxygen, the choice of such a value becomes somewhat arbitrary. Second, it might happen that the value for oxygen, even when determined by the investigator, was the most inaccurate of all his determinations; thus the accuracy of his other values would be diminished. This is the case, for example, at Geneva, where the value for oxygen has been determined with less precision than for the other gases.

These disadvantages disappear when all results are calculated to the weight of one liter of the gas under standard conditions. All the investigators, with the exception of Leduc, have recorded the exact volume of the balloons employed. Leduc's values may be calculated, since he has determined the weight of a liter of air at Paris.

There is, finally, another reason for expressing the density by the weight per normal liter, and that is the fact that the volume of the balloon can be calibrated more accurately than the weight, of a gas for comparison, can be determined in the same balloon. Leduc reports that after an interval of two years the capacity of a balloon originally found to be 2.27636 liters, was 2.27630 liters, or the variation was $1: 38,000$. At Geneva an accuracy of I part in 30,000 to 35,000 , between successive calibrations, has been easily realized. It is necessary to deternine the capacity of the balloon for water at $0^{\circ}$, and under these conditions no correction for the expansion of the glass is required. ${ }^{1}$

Choice of Final Values. - The following are some of the rules which have guided us in making a choice between the various values obtained for the density of each gas.

First, the nature of the method employed for the production and purification of the gas has been taken into consideration. This is, to our mind, the most important consideration in the density determinations. Those results showing concordance in the values obtained for the gas prepared in two or three different ways have, therefore, been given most weight.

${ }^{1}$ Difficulty was experienced in weighing the balloon filled with water at $0^{\circ}$, as the water expands when the balloon is placed on the balance. This has been overcome by attaching a bulb tube to the tubule of the balloon, by means of a ground joint or a rubber tubing, the whole apparatus having a counterpoise of the same glass and the same volume. 
A second consideration, equally important, is the concordance obtained by different investigators for the same gas. When the average values of two observers agree, while that of a third experimenter differs by a considerable amount, they have not been given equal weight, but the third value has been given less weight than the first two. Frequently, an experimenter has made a more or less accurate determination of the limit of error of his work. We have applied the correction in such a way as to bring the value nearer the mean of the other two observers. Similarly, when an error was discovered in the course of subsequent work, we have not taken into account that value known to be erroneous.

As will appear in the following discussion, while the preceding rules are suggested, an accurate choice is difficult to make. We would add that in abandoning several of Leduc's values, giving preference to others, generally more recent, in their stead, there has been no intention of diminishing the importance of Leduc's work. If the modern determinations have benefited by a more perfect technique, still we must recognize that to Leduc belongs the credit of having been the first to give a complete solution, theoretical and experimental, of the problem of the rigorous determination of the molecular weights of gases as a function of their densities.

\section{Discussion of Results.}

Oxygen.-The system of atomic and molecular weights being referred to oxygen $(\mathrm{O}=\mathrm{I} 6)$, the exact determination of the density of this gas is of considerable importance.

Morley (Z. physik. Chcm., 20, I (I896)) has made 4I determinations of this value, of which the averages of three series are:

\begin{tabular}{|c|c|c|c|c|}
\hline Series. & $\begin{array}{l}\text { No of deter- } \\
\text { minations. }\end{array}$ & $\begin{array}{l}\text { Weight of a stand- } \\
\text { ard liter. } \\
\text { Grams }\end{array}$ & $\begin{array}{r}\text { Capacity } \\
\text { balloon } \\
\text { Liters }\end{array}$ & \\
\hline 1 & 9 & $\mathrm{I} .42879$ & $2 \mathrm{I} 6$ and & 8.8 \\
\hline II. $(a)$ & $6 ?$ & $1+2887$ & 20.06 & $20 \cdot .56$ \\
\hline $\begin{array}{r}\text { III. (a) } \\
(b)\end{array}$ & $\begin{array}{cc}\ldots & 7 \\
\ldots & \text { I0 }\end{array}$ & I. 42917 & $8.83,16.52$ & 15.38 \\
\hline
\end{tabular}

In the first series the temperatures varied between $15^{\circ}$ and $20^{\circ}$, measured with mercury and air thermometers, the apparatus being connected with a manometer open to the air. In the second, the balloons were submerged in pounded ice, and the pressure measured by a differential manometer. In the third, the balloons were submerged in ice, the pressure being read on a barometer, the cistern of which communicated with the interior of the balloon.

The oxygen was prepared from potassium chlorate and by electrolysis of a solution of caustic potash for Series III $(b)$. The extreme variations between the individual observations were as follows:

$$
\begin{gathered}
4.8 \\
\text { I0,000 }
\end{gathered} \text { for Series I. } \frac{2}{10,000} \text { for Series II }(a), \begin{gathered}
6.9 \\
10,000
\end{gathered} \text { for Series II }(b), \frac{6.8}{10,000}
$$

for Series III $(a), \frac{7}{\operatorname{I0,000}}$ for Series III $(b)$. Referred to the averages, these extreme variations would be reduced to about one-half.

capacities are given in round numbers. 
Morley gives double weight to the average of Series III, and thus arrives at the value, I liter oxygen $=I .42900 \pm 0.000034$.

Rayleigh (Proc. Roy. Soc., 53, I44 (I893)) used oxygen prepared by three different methods: (a) by heating a mixture of potassium chlorate and sodium chlorate; (b) by heating potassium permanganate; $(c)$ by electrolysis of water, leading the gas thence through a column of copper oxide heated to redness. The balloon was filled at $0^{\circ}$ under a pressure of about I atm. at London, its capacity being $1836.52 \mathrm{cc}$. The averages of the results (weight of oxygen contained in the calibrated balloon) for each series are:

\begin{tabular}{|c|c|c|}
\hline $\begin{array}{l}\text { No. of experi- } \\
\text { ments. }\end{array}$ & $\begin{array}{l}\text { Weight of } \\
\text { oxygen. } \\
\text { Grams. }\end{array}$ & Extreme error. \\
\hline Chlorates $(a) \ldots \ldots \ldots$ & 2.6269 & $2 \cdot 3 / 10,000$ \\
\hline (b) $\ldots \ldots \ldots \ldots 5$ & 2.6269 & $2 \cdot 3 / 10,000$ \\
\hline Permanganate $\ldots \ldots \ldots 3$ & 2.6271 & $1.1 / 10,000$ \\
\hline Electrolysis $(a) \ldots \ldots \ldots$, I & $2.627 \mathrm{I}$ & $\ldots \ldots$ \\
\hline$\quad(b) \ldots \ldots \ldots 2$ & 2.6272 & $\ldots \ldots$ \\
\hline $\begin{array}{r}\text { Average, } \\
\text { Correction for contraction, }\end{array}$ & $\begin{array}{l}2.62704 \\
0.00056\end{array}$ & \\
\hline Corrected weight, & 2.62760 & \\
\hline
\end{tabular}

I liter of oxygen at Paris $=1.42952$.

Dividing the value by 1.00033 , the gravity factor, 1 normal liter of oxygen $=1.42905$ grams.

Leduc (Recherches sur les gaz, Paris, 1898), using the balloon method, has reported the density relative to that of air, at Paris. He prepared the oxygen $(a)$ by electrolysis of an aqueous solution of caustic potash or sulphuric acid, (b) by decomposition of potassium permanganate, (c) by electrolysis of dilute sulphuric acid, the gas then passing through a column of hot copper oxide.

By the method $(a)$ the values, referred to air, varied between I.ro5or and 1.10516 , average $=1.105 \mathrm{I}$, extreme variation $\mathrm{I} .4 / 10,000$. By the method $(b),{ }^{1}$ I.IO527; by the method (c) (3 determinations), I.1052I.

Rejecting the results for (a) Leduc adopts 1.10523 for the density of oxygen referred to air at Paris. From this the weight of the normal liter is $\frac{1.29316 \times 1.10523}{1.00033}=1.42876$ grams (taking into account the weight of a liter of air at Paris, determined by the same investigator to be 1.29316 , which he claims is accurate to $1 / 20,000$ ); or in round numbers, allowing for the accuracy of $\mathrm{I} / 20,000$ claimed by Leduc, $\mathrm{I}$ liter of oxygen $=1.4288$ grams.

Jaquerod and Pintza (Compt. rend., I39, I29 (I904)), working in this laboratory, have made five determinations of the density of oxygen, obtained by heating potassium permanganate, by filling a volumeter of about 3.5 liters capacity, at $0^{\circ}$ and about I atmosphere pressure. These determinations, showing an extreme difference of $6 / 10,000$, give for the weight of the standard liter

$$
\mathrm{I} \text { liter oxygen }=\mathrm{I} .4292 \text {. }
$$

${ }^{1}$ Leduc does not mention the number of determinations. It would appear that only one was made. 
These determinations were made by the volumeter method. The authors consider their results to be a little too high, and for their further calculations have adopted the value I.4290. ${ }^{1}$

Grav ( $J$. Chem. Soc., 87, I607 (190,5)), in the course of his work on the density of nitric oxide, has made six determinations of the density of oxygen, which show remarkable agrement among themselves. The gas was prepared by heating recrystallized potassium permanganate. The results give for the weight of a balloon of $267.43 \mathrm{cc}$. capacity, filled at $0^{\circ}$ and one atm. pressure at Bonn, the value 0.38228 , the extreme variation being only $1.6 / 10,000$. A correction brings the capacity of the balloon to $267.388 \mathrm{cc}$. so that the weight of a normal liter, taking into acconnt the factor for the gravity at Bonn, is

$$
\text { I liter of oxygen }=\frac{0.38228}{0.267388 \times 1.000505}=1.42896 \text { grams, }
$$

a value differing from that of Morley's by only $1 / 36,000$.

Résumé.--Morley's deterninations, being the most numerous, and cartied on under the most varied conditions, would appear to deserve the greatest weiglit, even though the individual variations are relatively large. Raylcigh's walte is $1 / 20,000$ greater than Morley's, while Gray's is $1 / 36,000$ less. On the other hand, the less accurate work of Leduc and Jaquerod and Pintza differ from this by $\mathrm{I} / 7000$, one greater and one less. Whether the mean of all five determinations, or of only the first three, which merit the greater conficlence because of the greater number of single determinations and the close agreement between the single determinations, be taken, the value is the same-r.42900. 'The variations -0.00005 (Rayleigh) and …c.00004 (Gray), from the mean of Morley's values, are of the sane orcler as the probable error calculated for the latter $(\div 0.000034)$.

The value, $\mathrm{I}$ liter of oxygen $=1.42900$, is therefore adopter, and is certainly correct to $\mathrm{I} / \mathrm{I} 0000$ and probably to $\mathrm{I} / 20000$.

Sitric Oxide.-Leduc (Séances soc. franc. physique, I893, p. 214 ) determined the density of the gas in 1893 , and reported it to be 1.0388 referred to air, from which the wcight of a normal liter can be calculated to be $1.3+20$. Leduc suppressed this value in his memoirs published in I 898; therefore, it can hardly be taken into account. It is evidently too high. During the investigation carried on with M. Davila we obtained very nearly the same value when using a gas which had not been purified by liquefaction and fractional distillation, so that it would appear that such a high value is due to the presence of traces of nitrous oxide in the gas.

Gray was the first to make an accurate determination of the density of this gas, using the balloon method. 'The gas was prepared by the action of acetic acid on sodium nitrite and potassium ferrocyanide, washed with caustic potasin, dried over phosphorts pentoxide, then liqueficd and fractionated at low temperatures. Six determinations,

D. Berthelot has recently reported all the density determinations made in this laboratory, referred to this unit 1.4292 (Compt. rend., 144,260 ). In view of the few determinations made, and the reservations made by the authors, this has diminished the accuracy of our other results. In all our other work we have adopted the value 1.4200 . See 1. chim. phis., 4, 3.3.3. 
showing an extreme difference of $3 / 10,000$, gave as the weight of the gas contained in the balloon, $0.3585 \mathrm{I}$. Six determinations of oxygen (extreme variation I.3/10,000) gave 0.38228 . Adopting Rayleigh's value for oxygen ( 1.42905 ) the value for nitric oxide is calculated.

$$
\text { I liter of nitric oxide }=\frac{\mathrm{I} .42905 \times 0.3585 \mathrm{I}}{0.38228}=\mathrm{I} .3402 \text { grams. }
$$

On the other hand, he calculates, from the capacity of the balloon, ${ }^{1}$ I liter of nitric oxide $=$ I.340I I and I liter of oxygen $=$ I.42896. Gray adopts the value $\mathrm{r} .3402$ grams. This practically conforms with that which would result if the value $\mathrm{O}_{2}=\mathrm{I} .42900$ were used.

$$
\text { I liter } \mathrm{NO}=\frac{1.4290 \times 0.3585 \mathrm{I}}{0.38228}=1.34015 \text {. }
$$

Guye and Davila have carried out three series of determinations on gas prepared from three different sources, $(a)$ by the action of ferrous sulphate on nitric acid; (b) by decomposition by means of mercury of a solution of sodium nitrite acidified with sulphuric acid; (c) by the action of sulphuric acid on a concentrated solution of sodium nitrite. The method of purification was in each case the same as that employed by Gray, with the exception that the washing with caustic potash was omitted, as it changes a part of the gas to nitrous oxide.

Weighings were made in two balloons of about 0.8 and 0.4 liter capacity, respectively.

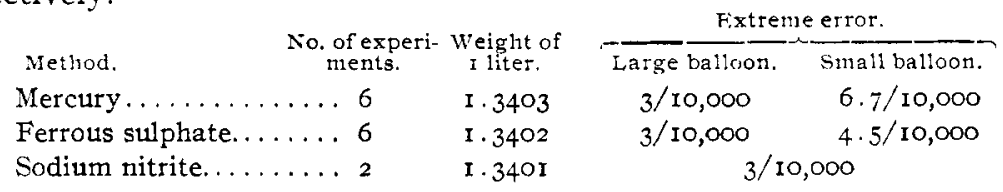

The mean of the I4 experiments, together with the mean for each method, gives I liter $\mathrm{NO}=1.3402$, a value identical with Gray's and which we hereby adopt.

Carbon Dioxide.-Rayleigh reports the density of this gas to be 1.52909 as referred to air. He gives no details as to the method, the number of determinations, or their probable error. The gas was prepared, $(a)$ by the action of hydrochloric acid on marble, (b) by the same acid on sodium carbonate.

Leduc obtained the mean value of $\mathrm{I} .52874$ for three very concordant determinations on a gas prepared by the action of hydrochloric acid on marble. He adopts the value 1.5288 , in view of the probable error duc to traces of air. He also observes that the work of Regnault points to the value 1.5290 .

The value for a normal liter can then be deduced as follows:

$$
\begin{aligned}
& \mathrm{I} \text { liter of } \mathrm{CO}_{2}=\mathrm{I} .29284 \times \mathrm{I} .52909=\mathrm{I} .9769 \text { (Rayleigh). } \\
& 1 \text { liter of } \mathrm{CO}_{2}=\mathrm{I} .29273 \times \mathrm{I} .5288=\mathrm{I} .9763 \text { (Leduc). }
\end{aligned}
$$

Guye and Pintza have determined the weight of a normal liter directly, with a gas generated by heating sodium bicarbonate, and have found I.9768, the mean of three experiments having an extreme difference of $0.8 / 10,000$. The value finally accepted,

$$
\text { I liter of } \mathrm{CO}_{2}=\mathrm{I} .9768 \text {, }
$$

${ }^{1}$ By private communication. 
agrees to about $1 / 20,000$ with Rayleigh's value; it gives a certain weight to the lower result of Leduc and is justified by a consideration of the probable error. In the course of the work at Geneva, the difficulty experienced in obtaining carbon dioxide entirely free from air has been constantly kept in mind, and the gas which was used in these determinations was entirely soluble in cautic potash.

Nitrous Oxide.-Leduc, Rayleigh, and Guye and Pintza have determined the density of nitrous oxide.

Leduc used the commercia $1^{1}$ liquefied gas, rectified by distillation. He obtained the densities, referred to air, of I.5304, I.5298, I.530I. Average, I.530I; extreme error, 3.9/10,000. Rayleigh made his first series of determinations in 1897 with a gas obtained by the decomposition of ammonium nitrate, and purified by dissolving in water-heating to expel the gas, then drying. Five determinations, with a probable error of $\mathrm{I} .7 / \mathrm{IO}, 000$, gave the mean value $\mathrm{I}$.5295I referred to air. In 1904 Rayleigh (Rayleigh, Proc. Roy. Soc., 74A, 18I) repeated his work, using the commercial liquefied gas, purified in the same way as above, and obtained practically the same result. He then purified the commercial gas by fractional distillation at the temperature of liquid air, till the density remained constant, and obtained, as the mean of three very concordant observations, with an extreme difference of $0.8 / 10,000$, the value 1.5297 .

Irom this last value of Rayleigh's and from Leduc's, the following values for the weight of the normal liter may be calculated:

$$
\begin{aligned}
& \text { I liter of } \mathrm{N}_{2} \mathrm{O}=\mathrm{I} .9780 \text { (Leduc), } \\
& \mathrm{I} \text { liter of } \mathrm{N}_{2} \mathrm{O}=1.9777 \text { (Rayleigh). }
\end{aligned}
$$

Guye and Pintza have determined the weight of the normal liter directly, using the volumeter method. The gas was prepared by the double decomposition of hydroxylamine sulphate and sodium nitrite. The average of three experiments, having an extreme variation of $2.8 / 10,000$, is

$$
\text { I liter of } \mathrm{N}_{2} \mathrm{O}=1.9774 \text { ( (inye and Pintza). }
$$

The impurities which might be found in the commercial nitrous oxide are nitrogen peroxide, air or nitrogen. The fractionation at low temperatures might not bring about a complete elimination of these impurities, ${ }^{2}$ or it might be that the decomposition of hydroxylamine sulphate did not furnish a gas entirely free from air, even though the evactiation of the apparatus was repeated several times. Under these conditions, and considering the good agreement of the separate determinations made by Rayleigh in I904, we would adopt for the weight of the normal liter,

$$
\text { I liter } \mathrm{N}_{2} \mathrm{O}=1.9777 \text { gramis, }
$$

which is the mean of the values obtained by Rayleigh, Leduc, Guye and Pintza.

Hydrochloric Acid (jas.--Leduc has determined the density of hydrochloric acid gas, produced by the action of sulphuric acid on sodium chloride, and dried over phosphorus pentoxide, to be 1.2692 as compared with air. There is no indication of the number of determinations or

(Obtained by heating ammonium nitrate.

The washing with alkalies, as practiced commercially, is not sufficient to remove the last traces of $\mathrm{NO}_{\mathrm{p}}$. 
their accuracy, although he considers the last decimal doubtful. A normal liter would be $1.29273 \times 1.2692=1.6407$ grams.

Guye and Gazarian have repeated this determination with a gas prepared in the same way, but further purified by liquefaction at the temperature of liquid air and fractionation at a low temperature. ${ }^{1}$ A preliminary series of four determinations gave a mean of 1.6398 , with an extreme variation of $9 / 10,000$.

Gray, who has undertaken a revision of the density of hydrochloric acid gas, reports a series of six determinations, with a probable error of $3.7 / 10,000$, of which the mean is, one normal liter $=1.6397$. This differs from the preceding by $\mathrm{I} / \mathrm{I} 6,000$. The mean of these two concordant values would be 1.63975 or, suppressing the last decimal, I.6398. This value is given only provisionally, until further determinations shall be announced.

Ammonia Gas.-Leduc used ammonia gas obtained from a commercial ammoniacal solution called "pure," which he dried over molten caustic potash. Without reporting the number or the accuracy of his determinations, he gives the value $0.597 \mathrm{I}$ as compared with air, or the weight of one normal liter,

$$
1.29273 \times 0.5971=0.7719 \text { gram. }
$$

Guye and Pintza have determined the weight of a standard liter, using commercial liquefied ammonia. Assuming that Leduc's high value was due to the presence of organic bases, these experimenters previously purified the gas by leading it over red-hot quicklime, to transform the nitrogen of the organic bases into ammonia. The gas was then collected as ammonium chloride, in this form was recrystallized, and was finally set free by warming the salt with lime, and dried over long columns of recently molten caustic potash. It was entirely soluble in sulphuric acid. Five determinations (using a volumeter of 3.5 liters capacity) with an extreme error of $3.9 / 10,000$ gave a mean value 0.77079 , or in round numbers, I normal liter $=0.7708$. Perman and Davies (Proc. Roy. Soc., 78A, 34 (1906)) have repeated this determination, both by the volumeter method, using a balloon of 1.7783 liters capacity, and by the balloon method, using a balloon of 0.50476 liter capacity. With the gas from a commercial ammoniacal solution, repeating Leduc's procedure, they have arrived at a value $0.77 \mathrm{I} 7$, almost identical with Leduc's, thus proving the necessity for removing the organic bases. They then purified the gas by three different methods; $(a)$ by the method of Guye and Pintza, (b) by decomposing with caustic potash, ammonium oxalate which had been ten times recrystallized, (c) by reduction of sodium nitrite, in a caustic soda solution, by aluminum. They obtained the following results:

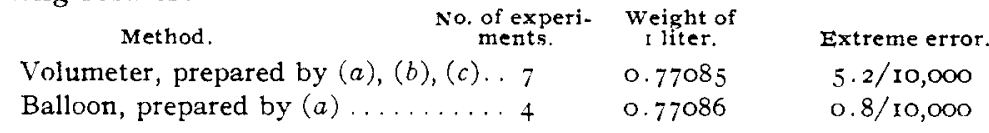

1 These fractionations were for the purpose of eliminating the volatile phosphorus compound, which, according to Richards and Wells (This Journal, 27, 459) is formed when hydrochloric acid passes over phosphorus pentoxide, and which would increase the density of the gas. Our work has also shown that the desiccation of the inside walls of the balloun is accomplished only after repeated rinsings with the hydrochloric acid gas. For these reasons Leduc's value must certainly be a little too high. 
The extreme error in the first series is undoubtedly due to the fact of the ammonia gas having been prepared by several methods. The authors adrise the value 0.77085 .

It appears from these experiments that Leduc's value cannot be considered in deciding upon a final value, as it is unquestionably affected by a constant error due to the presence of organic bases. The mean of the other three values is I liter of ammonia $=0.770837$, which can be taken in round numbers to be

$$
\text { I liter of ammonia }=0.7708 \text {, }
$$

differing from the above by $\mathrm{I} / \mathrm{I} 9,000$. It takes into account the fact that any source of error (presence of air or of organic bases, traces of moisture or condensation on the surface of the balloon) ${ }^{1}$ would tend toward raising the final value rather than lowering it. The mean of the leterminations by Perman and Davies, using the volumeter method, which they consider most accurate, and gas prepared by method $(a)$, is 0.77080 gram.

Sulphur Dioxide-Leduc reports 2.2639 , referred to air, as the mean of several deterninations (he does not state the number) having an extreme variation of $1.3 / 10,000$. The gas was prepared by the action of mereury on pure sulphuric acid.

Jaqueror and Pintza, using the volumeter method (capacity of balloon $=3.5$ liters) and a gas prepared by repeated fractional distillations of the liquefied sulphur dioxide of commerce, have obtained as the weight of one normal liter of sulphur dioxide 2.9266 grams, which represents the mean of seven determinations having an extreme difference of $1.7 / 10,000$.

This result agrees exactly with Leduc's,

$$
\text { I liter of sulphur clioxide }=1.29273 \times 2.2639=2.9266 \text {. }
$$

Baume, using two balloons of 0.3 and 0.5 liter capacity, respectively, las recently repeated this determination in the Geneva laboratory and obtained the same rest1t. The gas was purified by the same method as that employed by Jaquerod and Pintza. The extreme variation was $3.8 / 10,000$ for the large and $12.7 / 10,000$ for the small balloon. The mean of the two series is

$$
\text { I normal liter }=2.9266 \text {. }
$$

This, therefore, is the accepted value.

\section{Conclusions.}

'The various results have been tabulated as follows: In column I are the values obtained by Leduc; in column II those obtained by Rayleigh; in column III those of various other investigators (Morley, Ramsay, Gray, Perman and Davies); in column IV those obtained in the Geneva laboratory; in column $V$ the finally accepted value; in column VI the same value referred to the density of oxygen as one. 'This table has been com-

1 of the three nethods, $(a),(b),(c)$, the first would undoubtedly give the purest wats. 'lhe recrystallization of ammonium oxalate does not assure a complete elimination of organic bases, which is likewise true of the reduction of sodium nitrite by alumi. num, as the latter may contain snall amounts of carbon capable of being transformed into organic bases. In the course of my work with Pintza we proved conclusively that the ammonia gas obtained by the decomposition of magnesium nitride contained traces of organic hases. Its density was $1 / 1935$ higher than that of the gas prepared by methol a 
pleted by the addition of the better recent determinations of other gases ( $J$. chim. phys., 5, 203 (1907)):

WEIGHT OF NORMAL LITER (IN GRAMS).

\begin{tabular}{|c|c|c|c|c|c|c|}
\hline Gas. & Leduc & Rayleigh. & Others. & $\begin{array}{c}\text { Laboratory. } \\
\text { Guye. }\end{array}$ & $\begin{array}{l}\text { Accepted } \\
\text { value. }\end{array}$ & $\begin{array}{c}\text { Referred } \\
\text { to density } \\
\text { of } \mathrm{O}_{2} \text {. }\end{array}$ \\
\hline $\mathrm{O}_{2}$ & $(\mathrm{I} .4288)$ & I. 42905 & $\left\{\begin{array}{l}\mathrm{I} .42900 \mathrm{M} . \\
\mathrm{I} .42896 \mathrm{Gr}\end{array}\right.$ & $(\mathrm{I} .4292) \mathrm{J} . \mathrm{P}$. & I. 42900 & I.00000 \\
\hline $\mathrm{H}_{2}$ & 0.08982 & $(0.08998)$ & $0.089873 \mathrm{M}$ & $\cdots \cdots$ & 0.08987 & 0.062890 \\
\hline $\mathrm{N}_{2}$ & I. 2503 & 1.2507 & I. $2507 \mathrm{Gr}$ & $\ldots \ldots$ & I. 2507 & 0.87523 \\
\hline $\mathrm{CO}$ & I. 2501 & I. 2504 & $\ldots \ldots$ & $\ldots \ldots$ & I. 2504 & 0.87502 \\
\hline $\mathrm{NO}$ & $(1 \cdot 3429)$ & $\ldots \ldots$ & $\mathrm{I}: 3402 \mathrm{Gr}$ & I. 3402 G.D. & I $\cdot 3402$ & 0.93786 \\
\hline Ar & $\ldots \ldots$ & I. 7809 & I. $7808 \mathrm{R}$ & $\ldots \ldots$ & $\ldots \ldots$ & I. 2463 \\
\hline $\mathrm{CO}_{2}$ & I. 9763 & 1.9769 & $\ldots \ldots$ & I.9768 G.P. & I. 9768 & I. 3833 \\
\hline $\mathrm{N}_{2} \mathrm{O}$ & I. 9780 & I. 9777 & $\ldots \ldots$ & I. 9774 G.P. & I. 9777 & $I \cdot 3840$ \\
\hline $\mathrm{HCl}$ & $(1.6407)$ & $\ldots \ldots$ & $1.6397 \mathrm{Gr}$ & I.6398 G.G. & 1. 6398 & I. I 475 \\
\hline $\mathrm{NH}_{3}$ & $(0.7719)$ & $\ldots \ldots$ & 0.77085 P.D. & 0.7708 G.P. & 0.7708 & 0.53940 \\
\hline $\mathrm{SO}_{2}$ & 2.9266 & & & $\left\{\begin{array}{l}2.9266 \mathrm{~J} . \mathrm{P} \\
2.9266 \mathrm{~B}\end{array}\right.$ & 2.9266 & 2.0480 \\
\hline Air & I. 2927 & I. 2928 & $\ldots \ldots$ & $\ldots \ldots$ & 1.2928 & 0.90469 \\
\hline
\end{tabular}

${ }^{1}$ Dapier, J. chim. phys., 5, 203 and Arch. sci. phys. nat. [4], 24, 34.

The conclusions to be drawn from the work carried on at Geneva during the last three years, compared with that accomplished in other laboratories, are as follows:

I. The method gives results generally agreeing to at least I/Io,ooo, when one takes the mean of a half dozen determinations, the extreme difference between any two of which is not more than $3 / 10,000$ or $5 / 10,000$.

2. This agreement may be obtained even with gases as difficultly dried and purified as ammonia, hydrochloric acid, sulphur dioxide or nitric oxide. For these it is only necessary that the balloon be well dried with dry air, and then rinsed several times in succession with the dry gas which is to be studied. Under these conditions there is no appreciable difference between the mean obtained for a balloon of 3.5 liters capacity and one of 0.5 liter. The surface action is then negligible in work of an accuracy of the order of $1 / 10,000$.

3. The purification of the gas is especially important. The method based on the liquefaction of the gas, followed by distillation at low temperature, is recommended.

4. It is preferable to calculate directly the weight of a liter of the gas under standard conditions, rather than to report the density as compared with that of another gas (oxygen or air). By calibrating the balloon at $0^{\circ}$, its volume may be determined to $1 / 30,000$ - an accuracy which could be approached, in the density determination, only by a very considerable number of determinations.

5. The most probable values for the density of the gases, determined with an accuracy of the order of $\mathrm{I} / \mathrm{I} 0, \infty 00$, are collected in columns $\mathrm{V}$ and VI of the last table. 